\title{
Influencing mechanism of magnolol on expression of BDNF and Bax in rats with cerebral ischemic stroke
}

\author{
ZHENBO LIU $^{1 *}, \mathrm{JUN}^{\mathrm{XIE}}{ }^{2 *}, \mathrm{KAI} \mathrm{LIN}^{3}$ and LIGUO QI ${ }^{4}$ \\ ${ }^{1}$ The Second Department of Neurosurgery, Xingtai People's Hospital, Xingtai, Hebei 054031; \\ ${ }^{2}$ Department of Neurosurgery, Tongchuan People's Hospital, Tongchuan, Shaanxi 727000; \\ ${ }^{3}$ Department of Neurosurgery, Liaocheng People's Hospital, Liaocheng, Shandong 252004; \\ ${ }^{4}$ Department of Neurosurgery, Taian City Central Hospital, Taian, Shandong 271000, P.R. China
}

Received January 3, 2018; Accepted July 26, 2018

DOI: $10.3892 /$ etm.2018.6807

\begin{abstract}
The impact of magnolol on cerebral ischemic stroke in rats and the molecular mechanism were explored. Sprague-Dawley rat models were studied. Cerebral indexes, hematoxylin and eosin staining, TUNEL staining assay, reverse transcription-polymerase chain reaction (RT-PCR) and western blotting were applied. The cerebral index in model group was significantly higher than that in sham operation group, and the cerebral index was obviously decreased after magnolol administration. Inflammatory cells accumulated in the brain tissue of rats in the model group. Abundant apoptotic cells were produced in the model group, which was overtly improved after rats were given magnolol. RT-PCR and western blot analysis showed that expression of mRNA and protein of brain-derived neurotrophic factor (BDNF) were distinctly decreased in model group, and increased after rats were given magnolol; while mRNA and protein expression of $\mathrm{Bcl}-2$ associated $\mathrm{X}$ protein (Bax) were significantly raised in model group, and reduced after rats were given magnolol. The results showed that there were statistically significant differences in expression of BDNF and Bax among sham operation, model and magnolol administration groups $(\mathrm{p}<0.01)$. In conclusion, magnolol can increase the expression of BDNF and decrease the expression of Bax, thereby inhibiting apoptosis to protect the nerves, and magnolol can improve cerebral ischemic stroke in rats.
\end{abstract}

Correspondence to: Dr Liguo Qi, Department of Neurosurgery, Taian City Central Hospital, 29 Longtan Road, Taian, Shandong 271000, P.R. China

E-mail: wayishan2@163.com

${ }^{*}$ Contributed equally

Key words: magnolol, cerebral ischemic stroke, BDNF, Bax

\section{Introduction}

Cerebral ischemic stroke is a generic term for cerebral tissue necrosis caused by cerebral supplying artery stenosis or occlusion or insufficient cerebral blood supply. As the pace of life speeds up, and as the way of life has changed dramatically, the incidence rate of cerebral ischemic stroke is increasing year by year, seriously affecting the quality and threatening life $(1,2)$. Therefore, it is urgent to find effective methods and drugs for the treatment of cerebral ischemic stroke. With the development of science of Chinese traditional medicine, people increasingly focus on the study of natural products. Because traditional Chinese medicine has the advantages of high efficiency, low toxicity and multiple targets, work is being carried out to finding an effective anti-cerebral ischemic stroke drug using traditional Chinese medicine (3). Magnolol is a natural product with very high activity extracted from Chinese traditional medicine. It has good lipid solubility, so it can penetrate the blood-brain barrier, enter into and work in the brain. Previous studies have confirmed that magnolol has effects of anti-inflammation, anti-virus, antitumor, and inhibition of apoptosis, but magnolol's anti-ischemic stroke effect is still undetermined (4-6). In this study, by constructing models of rat with cerebral ischemic stroke and after administration of magnolol, the effect of magnolol on rats with cerebral ischemic stroke was observed and the molecular mechanism was studied, to deeply investigate the action mechanism of magnolol.

\section{Materials and methods}

Experimental animals and grouping. A total of 60 male

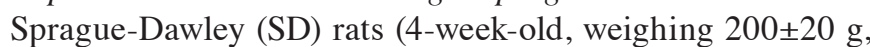
purchased from Beijing Vital River Laboratory Animal Technology Co., Ltd., Beijing, China) were selected and randomly divided into sham operation, model and magnolol administration groups, with 20 rats in each group. The rats were kept in cage with controlled temperature and light cycles $\left(24^{\circ} \mathrm{C}\right.$ and $12 / 12$ light cycles $)$ and free access to water and food. The humidity was $60 \pm 10 \%$. The rats in magnolol administration group were intraperitoneally injected with magnolol $(75 \mathrm{mg} / \mathrm{kg}$ ) for 7 consecutive days, once per day. 
After 7 days of administration, the rats were fasted for $12 \mathrm{~h}$ but were given drinking water. Middle cerebral artery occlusion (MCAO) reperfusion models of rat were established in model and magnolol groups by longa's animal model. The study was approved by the Ethics Committee of Xingtai People's Hospital (Xingtai, China).

Main reagents. A terminal-deoxynucleotidyl transferase mediated nick end-labeling (TUNEL) kit (Roche, Basel, Switzerland); a bicinchoninic acid (BCA) protein quantification kit (Beyotime Biotechnology, Shanghai, China); a TRIzol total ribonucleic acid (RNA) extraction kit and a reverse transcription-polymerase chain reaction (RT-PCR) reverse transcription kit (both from Tiangen Biotech, Beijing, China); and glyceraldehyde phosphate dehydrogenase (GAPDH), antibrain-derived neurotrophic factor (BDNF) and Bcl-2-associated $\mathrm{X}$ protein (Bax) monoclonal antibodies (ProteinTech Group, Inc.; Wuhan Sanying Biotechnology; Wuhan, China).

\section{Experiment methods}

Determination of relative cerebral index. The rats in each group were weighed and the body weight was recorded. Then, the rats were sacrificed and the brain was quickly removed to record the brain weight. The cerebral index was calculated by: Cerebral index $=$ brain weight/body weight.

Hematoxylin and eosin $(H \& E)$ staining. Paraffin-embedded rat brain sections were routinely dewaxed until the paraffin was replaced by water and then rinsed with phosphate-buffered saline (PBS) (3 min/time x 3 times). Then, sections were stained with hematoxylin for $5 \mathrm{~min}$, de-stained with $1 \%$ hydrochloric acid $(\mathrm{HCl})$, and rinsed with double distilled water $(5 \mathrm{~min} /$ times x6 times). After that, sections were made blue with lithium carbonate saturated solution for 1 to $2 \mathrm{~min}$ and washed with double distilled water (5 min/times x3 times). Color separation was then done with $80 \%$ alcohol and rinsing was conducted with double distilled water ( 5 min/times x 3 times). Later, sections were stained with eosin for $5 \mathrm{~min}$ and rinsed with double distilled water (5 min/times $\mathrm{x} 3$ times). Last, sections were dewatered, hyalinized, blocked with resin, and photographed using an upright microscope (Olympus Corporation, Tokyo, Japan).

Detection of apoptosis. The detection was performed in accordance with the instructions of the TUNEL kit. Paraffin-embedded sections were routinely dewaxed until the paraffin was replaced by water, washed with $10 \mathrm{mM}$ PBS for $3 \mathrm{~min} /$ time $\mathrm{x} 3$ times, incubated in $1 \% \mathrm{H}_{2} \mathrm{O}_{2}$ at room temperature for $20 \mathrm{~min}$ to inhibit endogenous peroxidase, rinsed with PBS for $3 \mathrm{~min} / \mathrm{time} \mathrm{x} 3$ times, digested with $20 \mu \mathrm{g} / \mathrm{ml}$ protease $\mathrm{K}$ for $20 \mathrm{~min}$ at $37^{\circ} \mathrm{C}$, washed with PBS for $3 \mathrm{~min} /$ time $\mathrm{x} 3$ times, incubated with terminal deoxynucleotidyl transferase (TdT) buffer for $10 \mathrm{~min}$, immersed in TUNEL mixture [100 $\mu 1$ mixture containing $1 \mu \mathrm{l} \mathrm{TdT}$ and $1 \mu \mathrm{l}$ solution of biotin-epsilon-aminocaproyl-[5-\{3-aminoallyl $\}-2 '-d e o x y u r i d i n e-5 '-t r i p h o s p h a t e]$ (Biotin-11-dUTP)] at $37^{\circ} \mathrm{C}$ for $90 \mathrm{~min}$, washed with PBS for $3 \mathrm{~min} /$ time $\mathrm{x} 3$ times, and placed in (Tris-HCI buffer) TB for $15 \mathrm{~min}$ at room temperature to terminate the reaction. Then, sections were dropwise added with anti-Avidin-horseradish peroxidase (HRP) solution and incubated at $37^{\circ} \mathrm{C}$ for $1 \mathrm{~h}$,
Table I. RT-PCR primer sequences for BDNF, Bax and GAPDH mRNAs.

Gene names $\quad$ Primer sequences

\begin{tabular}{ll} 
BDNF & F: 5'-3' GCCCATATGACCATCCTTTTCCTTA \\
& R: 3'-5' CTATCTTCCCCTTTTAATGGTCAGT \\
Bax & F: 5'-3' CAGGATGCGTCCACCAAGAA \\
& R: 3'-5' CGTGTCCACGTCAGCAATCA \\
GAPDH & F: 5'-3' GAGCCGGGAAATCGTGCGT \\
& R: 3'-5' GGAAGGAAGGCTGGAAGATG \\
\hline
\end{tabular}

BDNF, brain-derived neurotrophic factor; GAPDH, glyceraldehyde phosphate dehydrogenase; Bax, Bcl-2-associated X protein; mRNAs, messenger RNAs.

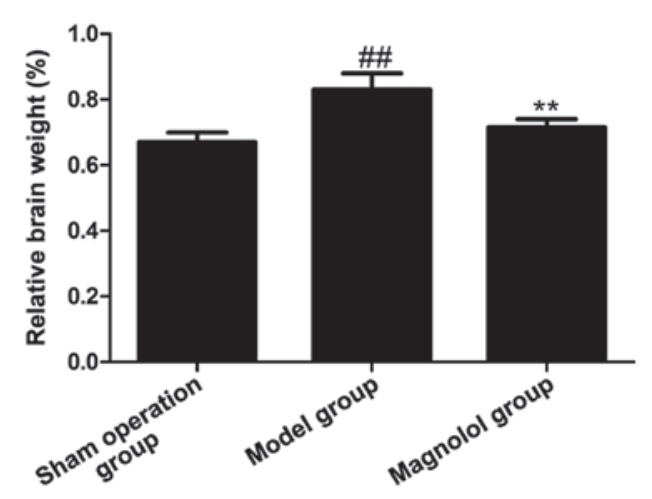

Figure 1. Cerebral index of in sham operation, model and magnolol groups. Compared with sham operation group, ${ }^{\#} \mathrm{p}<0.01$; compared with model group, ${ }^{* *} \mathrm{p}<0.01$.

rinsed with PBS for $3 \mathrm{~min} /$ time $\mathrm{x} 3$ times, subjected to color development with diaminobenzidine (DAB) for 20 to $30 \mathrm{~min}$, and rinsed to terminate the reaction. Conventional dehydrating, hyalinizing and blocking with resin were carried out. Three fields were observed randomly under high power lens (x200 magnification), and the mean value was calculated as the number of apoptotic cells in the animal.

RT-PCR analysis. Moderate amount of brain tissues in sham operation, model and magnolol groups were transferred into $1 \mathrm{ml}$ TRIzol reagent and fully ground into homogenate. The homogenate was left to stand for $5 \mathrm{~min}$ at room temperature and the sample was completely lysed using Tissuelyser (Qiagen GmbH, Hilden, Germany). The homogenate was centrifuged at $12,000 \mathrm{x}$ g for $5 \mathrm{~min}$ at $4^{\circ} \mathrm{C}$, and the supernatant was carefully collected, added with chloroform, mixed well, and placed at room temperature for $5 \mathrm{~min}$. The supernatant was carefully collected after centrifugation at $12,000 \mathrm{x} \mathrm{g}$ for $15 \mathrm{~min}$ at $4^{\circ} \mathrm{C}$, added with the same volume of isopropanol, left to stannd at room temperature for $10 \mathrm{~min}$, and centrifuged at $12,000 \mathrm{x} \mathrm{g}$ for $10 \mathrm{~min}$ at $4^{\circ} \mathrm{C}$, and the precipitate was then collected. Ethanol (75\%) was added, with homogeneous mixing, to wash the RNA precipitation. Later, RNase-free water was added to completely dissolve it. The ratio of optical density (OD) 260/OD280 and the RNA concentration were 

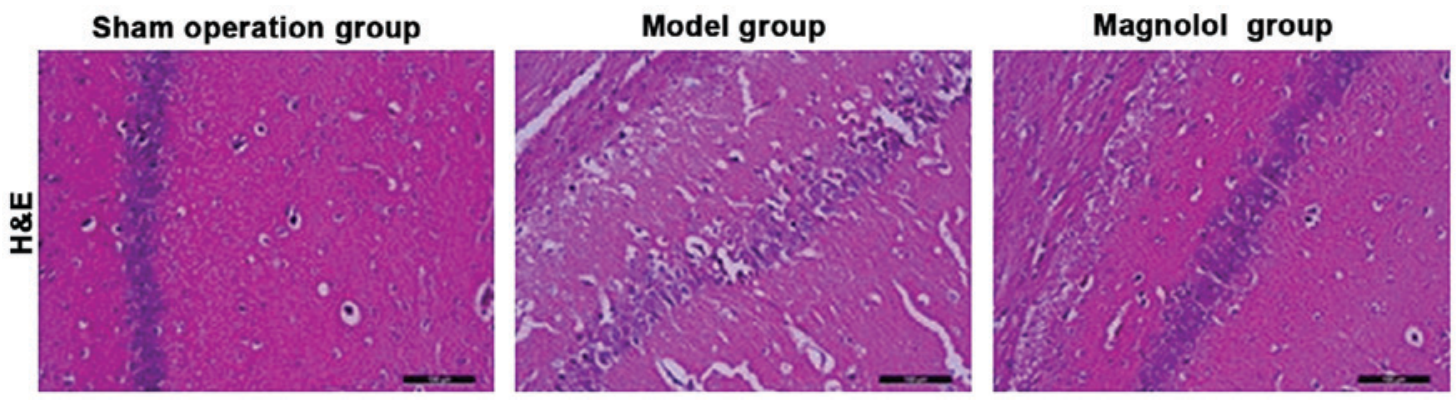

Figure 2. H\&E staining in brain tissues of rats in sham operation, model and magnolol groups (x200 magnification).

then measured. Finally, the reaction product was subjected to RT-PCR analysis via stepwise amplification performed based on the primer sequence templates shown in Table I.

\section{Western blot analysis}

Protein extraction. Brain tissues of rats in each group were collected and washed twice with frozen saline, respectively. Protein extraction was run according to the instructions of the total protein extraction kit. The tissue was added with lysate, homogenized for $1 \mathrm{~min}$ by a homogenizer and centrifuged at $12,000 \mathrm{x} \mathrm{g}$ for $10 \mathrm{~min}$ at $4^{\circ} \mathrm{C}$. Then, the supernatant, i.e., total protein in liver tissue, was collected. The protein concentration was determined by the BCA protein concentration kit and the supernatant was sub-packaged and stored at $-80^{\circ} \mathrm{C}$ until use.

Protein denaturation. The total protein extract was mixed evenly with $2 \mathrm{X}$ loading buffer based on the volume ratio of 1:1, heated in boiling water for $5 \mathrm{~min}$, cooled naturally, and stored at $4^{\circ} \mathrm{C}$ until use.

Sodium dodecyl sulfate-polyacrylamide gel electrophoresis (SDS-PAGE). SDS-PAGE separation gel in an appropriate proportion was prepared according to the molecular weight of target protein, with a coagulation of $\sim 1 \mathrm{~h}$. Then, 5\% SDS-PAGE spacer gel was prepared and coagulated for $\sim 0.5 \mathrm{~h}$. Electrophoresis buffer was added, and the denatured protein samples were loaded onto sample loading wells according to the protein concentration, so that the total protein content per well was the same. Electrophoresis was performed at a constant voltage of $220 \mathrm{~V}$ and stopped after bromophenol blue reached the bottom of the gel.

Membrane transfer. The gel was cut based on the molecular weight of target protein, and put into transfer buffer. And 1 layer of polyvinylidene fluoride (PVDF) membrane and 6 layers of filter paper were cut according to the size of the gel. The PVDF membrane was firstly immersed in methanol for $10 \mathrm{sec}$, and then put into the transfer buffer together with the filter paper. They were put into a transfer unit in the following sequence: Positive electrode, three layers of filter paper, PVDF membrane, gel, three layers of filter paper and negative electrode, with attention paid to edge alignment so as to prevent blistering. The membrane transfer was for $2 \mathrm{~h}$ at a constant voltage of $110 \mathrm{~V}$.

Blocking. The PVDF membrane containing protein was placed and blocked in 5\% skimmed milk on a shaker at room temperature for $2 \mathrm{~h}$.

Immune response. The blocked membrane was washed with Tween/Tris-buffered saline (TTBS) for $5 \mathrm{~min}$, placed into the mouse anti-rat BDNF, Bax and GAPDH primary monoclonal antibodies (1:1,000; cat. nos. 66292-1-Ig, 60267-1-Ig and 60004-1-Ig, respectively; ProteinTech Group, Inc.; Wuhan Sanying Biotechnology) in corresponding proportion, and incubated overnight at $4^{\circ} \mathrm{C}$. The membrane was washed with TTBS 3 times (10 min/time), put into the corresponding goat anti-mouse secondary polyclonal antibody $(1: 1,000$; cat. no. SA00001-1; ProteinTech Group, Inc.; Wuhan Sanying Biotechnology), incubated on a shaker at room temperature for $3 \mathrm{~h}$, and rinsed with TTBS 3 times (10 min/time).

Enhanced chemiluminescence (ECL). A gel imager was preheated for $30 \mathrm{~min}$. Reagents A and B in the ECL kit were mixed evenly at same volume, added dropwise onto the PVDF membrane, with full contact, kept in the dark and subjected to color development for $1 \mathrm{~min}$. The filter paper was used to blot up excess liquid around the membrane, and then the membrane was placed into the gel imager. Later, dynamic integration mode was applied to take pictures, and the results were observed. Image analysis software was employed for image analysis.

Statistical analysis. Experimental data are expressed as mean \pm standard error of the mean (mean \pm SEM). For experimental results, Statistical Product and Service Solutions (SPSS) 17.0 statistical software (SPSS, Inc., Chicago, IL, USA) was utilized for statistical analysis. The t-test was used for mean comparison between the two groups. One-way analysis of variance (ANOVA) was applied for comparison of sample average among multiple groups and the post hoc test was SNK test. $\mathrm{P}<0.05$ was considered to indicate a statistically significant difference.

\section{Results}

Effect of magnolol on cerebral index. As shown in Fig. 1, the cerebral index in model group was increased significantly compared with that in sham operation group, while the cerebral index was obviously lower in magnolol administration group than that in model group. This result showed that magnolol can effectively affect cerebral index and improve brain injuries.

Results of $H \& E$ staining. H\&E stained brain tissue sections in sham operation, model and magnolol groups were used to find differences in pathological features among samples. According to Fig. 2, compared with the brain tissue section of rats in sham group, a large number of inflammatory cells were 

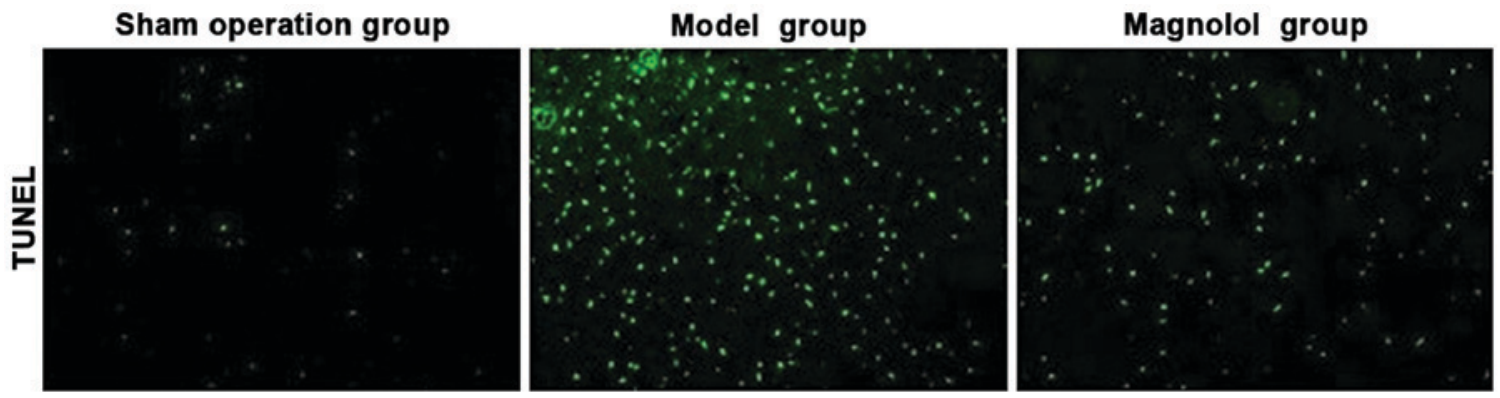

Figure 3. TUNEL staining in brain tissues of rats in sham operation, model and magnolol groups (x200 magnification).
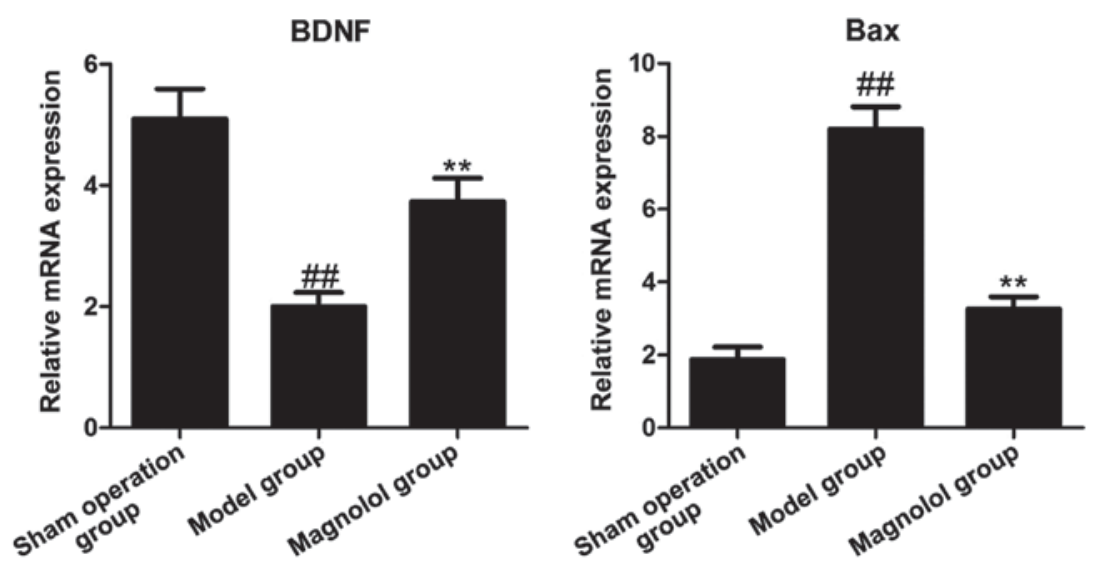

Figure 4. mRNA expression of BDNF and Bax in brain tissues of rats in sham operation, model and magnolol groups. Compared with sham operation group, ${ }^{\#} \mathrm{p}<0.01$; compared with model group, ${ }^{* *} \mathrm{p}<0.01$. BDNF, brain-derived neurotrophic factor; Bax, Bcl-2-associated X protein.
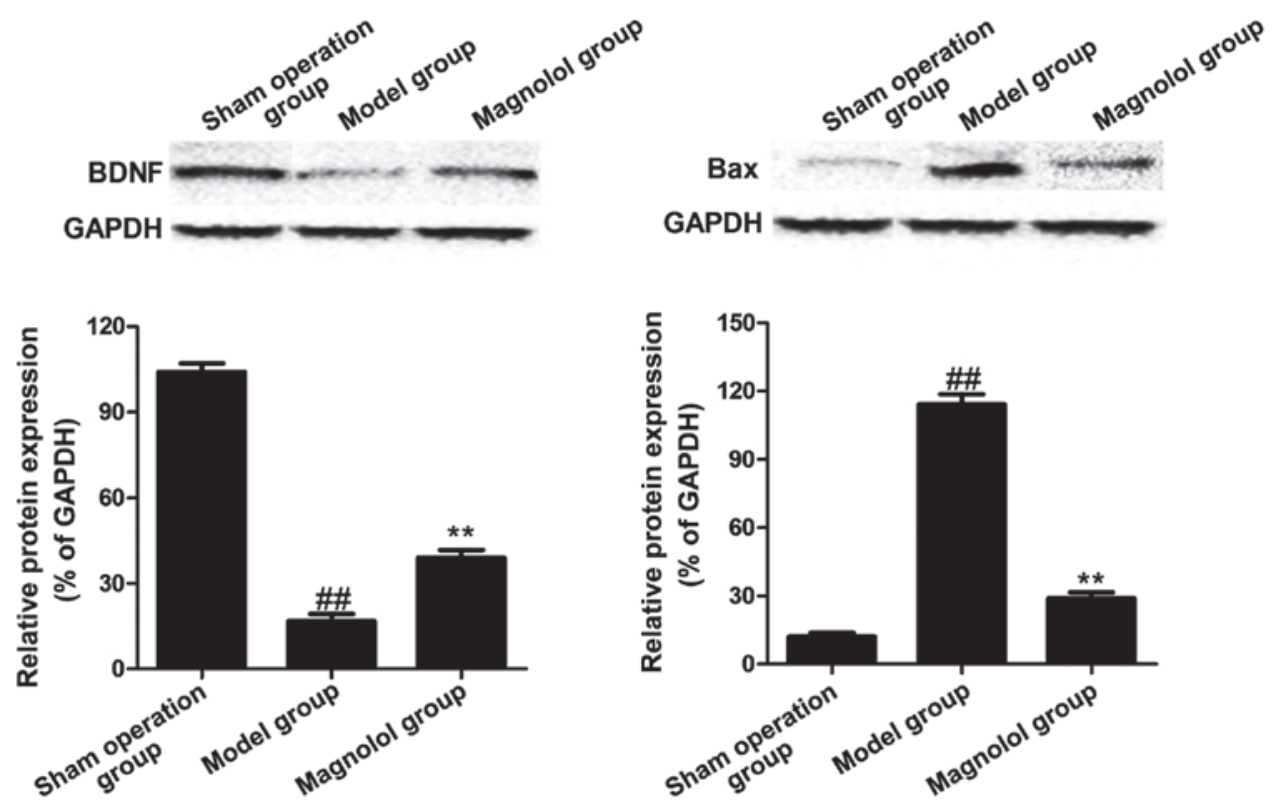

Figure 5. Protein expression levels of BDNF and Bax of rats in sham operation, model and magnolol groups. Compared with sham operation group, ${ }^{\# \#} \mathrm{p}<0.01$; compared with model group, ${ }^{* *} \mathrm{p}<0.01$. BDNF, brain-derived neurotrophic factor; Bax, Bcl-2-associated X protein.

found in the brain tissue section of rats in model group, with pyknotic, broken or even disappeared cell nuclei, and severe brain injuries; after rats were given magnolol, the inflammatory cells were overtly reduced, and the nuclei were more complete, suggesting that magnolol can improve brain injuries.
Results of TUNEL staining. The apoptosis of brain tissue of rats in sham operation, model and magnolol groups was observed through TUNEL staining. As shown in Fig. 3, compared with the brain tissue of rats in sham operation group, the brain tissue of rats in model group had abundant apoptotic cells, but 
magnolol group had distinctly reduced apoptotic cells after the administration of magnolol.

Results of RT-PCR. Total RNAs were extracted from brain tissue samples of rats in sham operation, model and magnolol groups. Through RT-PCR, it was found that BDNF was significantly decreased and Bax was clearly increased in the model group, which were effectively reversed after rats were given magnolol, indicating that magnolol has a neuroprotective effect and can inhibit the production of apoptosis (Fig. 4).

Protein expression levels of BDNF and Bax of rats in sham operation, model and magnolol groups. Western blotting results showed protein expression levels of BDNF and Bax of rats in sham operation, model and magnolol groups. As shown in Fig. 5, sham operation group had a high protein expression of BDNF but a low protein expression of Bax; while model group showed a low protein expression of BDNF but a high protein expression of Bax; in magnolol group, the protein expression of BDNF was significantly higher than that in model group, and the protein expression of Bax was clearly decreased compared with that in the model group.

\section{Discussion}

Brain is a major part of the higher neural system of the vertebrate and a higher nervous center to control movements, produce sensations and achieve high brain functions (7). Cerebral ischemic stroke seriously affects human health. Cerebral ischemic stroke refers to the sudden onset of blood flow perfusion reduction or complete obstruction of blood flow in supplying arteries in local brain tissues, leading to stopping of blood supply, oxygen supply, sugar supply, thereby resulting in disintegration and damage of local brain tissues (8-10). At present, methods used for the treatment of cerebral ischemic stroke are based on the improvement of blood circulation of the brain, increase in blood and oxygen supply in the penumbra area of ischemic area, control of brain edema, and prevention and treatment of complications. Although therapeutic methods for cerebral ischemic stroke are numerous, with up to dozens of drugs, the ideal therapeutic regimen is still controversial and uncertain (11). Therefore, the development of new drugs with special effects is necessary. However, new drugs in research and development mainly are chemical synthetic drugs, with high development costs, great risks, long cycles, and low success rates, so the annual number of new drugs in research and development is extremely limited.

Active constituents extracted from traditional Chinese medicine, especially Chinese herbal medicine, are not only important sources for new drug development (12-14), but also important means in preventing and treating modern diseases. Moreover, active constituents also have a great significance for the modernization and internationalization of Chinese traditional medicine (15). In recent years, natural products with significant anti-cerebral ischemic stroke effect have been continuously found (16). A large number of studies have shown that BDNF and Bax (an apoptosis-related factor) play important roles in cerebral ischemic stroke. This study took BDNF and Bax as targets to find effective Chinese traditional medicine. Magnolol is a natural compound; modern studies have indicated that magnolol has various pharmacological effects, such as antitumor, anti-hyperlipidemia, and antifungal effects; many previous studies have also revealed that magnolol has effects of anti-inflammation, anti-apoptosis and immunoregulation; however, the effect, mechanism of action, and target of action of magnolol on cerebral ischemic stroke are still unknown (17-20). Therefore, these unknown fields were studied in depth by the authors.

Male SD rats were studied and they were randomly divided into sham operation, model and magnolol administration groups. The cerebral indexes of each group were detected, respectively. The histopathological differences among each group were detected by H\&E staining. Apoptosis of each group was measured via TUNEL staining. The expression of mRNA and protein of BDNF and Bax in sham operation, model and magnolol groups was detected through RT-PCR and western blot analysis. The results showed that the cerebral index was dramatically decreased after the administration of magnolol. The results of H\&E staining revealed that a large number of inflammatory cells were found in the brain tissue of rats in model group, and the structure of cell nucleus was destroyed; magnolol was effective in improving brain injuries. The results of TUNEL staining indicated that plenty of apoptotic cells were produced in model group, and it was obviously improved after rats were given magnolol. RT-PCR and western blotting, respectively confirmed that in model group, the expression of mRNA and protein of BDNF was clearly decreased but distinctly raised after the administration of magnolol; the expression of mRNA and protein of Bax was overtly increased, but clearly declined after the administration of magnolol. This study shows that magnolol can improve cerebral ischemic stroke in rats; this is because magnolol can increase the expression of BDNF and decrease the expression of Bax. Magnolol, an anti-apoptotic neuroprotective drug, provides a new regimen for the prevention and treatment of cerebral ischemic stroke.

\section{Acknowledgements}

Not applicable.

\section{Funding}

No funding was received.

\section{Availability of data and materials}

The datasets used and/or analyzed during the current study are available from the corresponding author on reasonable request.

\section{Authors' contributions}

ZL and LQ performed PCR and western blot analysis. JX assisted in the detection of apoptosis. All authors read and approved the final manuscript.

\section{Ethics approval and consent to participate}

The study was approved by the Ethics Committee of Xingtai People's Hospital (Xingtai, China). 


\section{Patient consent for publication}

Not applicable.

\section{Competing interests}

The authors declare that they have no competing interests.

\section{References}

1. Nagai N, Kawao N, Okada K, Ishida C, Okumoto K, Ueshima S, Suzuki Y, Umemura K and Matsuo O: Initial brain lesion size affects the extent of subsequent pathophysiological responses. Brain Res 1322: 109-117, 2010.

2. Arai K, Jin G, Navaratna D and Lo EH: Brain angiogenesis in developmental and pathological processes: Neurovascular injury and angiogenic recovery after stroke. FEBS J 276: 4644-4652, 2009.

3. DiStefano PS, Friedman B, Radziejewski C, Alexander C, Boland P, Schick CM, Lindsay RM and Wiegand SJ: The neurotrophins BDNF, NT-3, and NGF display distinct patterns of retrograde axonal transport in peripheral and central neurons. Neuron 8: 983-993, 1992

4. Amin AR, Kucuk O, Khuri FR and Shin DM: Perspectives for cancer prevention with natural compounds. J Clin Oncol 27: 2712-2725, 2009.

5. Lin SY, Liu JD, Chang HC, Yeh SD, Lin CH and Lee WS: Magnolol suppresses proliferation of cultured human colon and liver cancer cells by inhibiting DNA synthesis and activating apoptosis. J Cell Biochem 84: 532-544, 2002.

6. Hwang ES and Park KK: Magnolol suppresses metastasis via inhibition of invasion, migration, and matrix metalloproteinase-2/-9 activities in PC-3 human prostate carcinoma cells Biosci Biotechnol Biochem 74: 961-967, 2010.

7. Chen J, Zhang C, Jiang H, Li Y, Zhang L, Robin A, Katakowski M, $\mathrm{Lu} \mathrm{M}$ and Chopp M: Atorvastatin induction of VEGF and BDNF promotes brain plasticity after stroke in mice. J Cereb Blood Flow Metab 25: 281-290, 2005.

8. Madinier A, Bertrand N, Mossiat C, Prigent-Tessier A, Beley A, Marie C and Garnier P: Microglial involvement in neuroplastic changes following focal brain ischemia in rats. PLoS One 4: e8101, 2009.
9. Mattson MP: Glutamate and neurotrophic factors in neuronal plasticity and disease. Ann N Y Acad Sci 1144: 97-112, 2008

10. Kawamoto Y, Nakamura S, Nakano S, Oka N, Akiguchi I and Kimura J: Immunohistochemical localization of brain-derived neurotrophic factor in adult rat brain. Neuroscience 74 : 1209-1226, 1996

11. Jean YY, Lercher LD and Dreyfus CF: Glutamate elicits release of BDNF from basal forebrain astrocytes in a process dependent on metabotropic receptors and the PLC pathway. Neuron Glia Biol 4: 35-42, 2008.

12. Gordaliza M: Natural products as leads to anticancer drugs. Clin Transl Oncol 9: 767-776, 2007.

13. Kong CW, Tsai K, Chin JH, Chan WL and Hong CY: Magnolol attenuates peroxidative damage and improves survival of rats with sepsis. Shock 13: 24-28, 2000.

14. Lee YM, Hsiao G, Chen HR, Chen YC, Sheu JR and Yen MH: Magnolol reduces myocardial ischemia/reperfusion injury via neutrophil inhibition in rats. Eur J Pharmacol 422: 159-167, 2001.

15. Teng CM, Ko FN, Wang JP, Lin CN, Wu TS, Chen CC and Huang TF: Antihaemostatic and antithrombotic effect of some antiplatelet agents isolated from Chinese herbs. J Pharm Pharmacol 43: 667-669, 1991.

16. Ho KY, Tsai CC, Chen CP, Huang JS and Lin CC: Antimicrobial activity of honokiol and magnolol isolated from Magnolia officinalis. Phytother Res 15: 139-141, 2001

17. Hamasaki Y, Kobayashi I, Zaitu M, Tsuji K, Kita M, Hayasaki R, Muro E, Yamamoto S, Matsuo M, Ichimaru T, et al: Magnolol inhibits leukotriene synthesis in rat basophilic leukemia-2H3 cells. Planta Med 65: 222-226, 1999.

18. Bang KH, Kim YK, Min BS, Na MK, Rhee YH, Lee JP and Bae KH: Antifungal activity of magnolol and honokiol. Arch Pharm Res 23: 46-49, 2000.

19. Wang JP, Hsu MF, Raung SL, Chen CC, Kuo JS and Teng CM: Anti-inflammatory and analgesic effects of magnolol. Naunyn Schmiedebergs Arch Pharmacol 346: 707-712, 1992.

20. Kuo DH, Lai YS, Lo CY, Cheng AC, Wu H and Pan MH: Inhibitory effect of magnolol on TPA-induced skin inflammation and tumor promotion in mice. J Agric Food Chem 58: 5777-5783, 2010 .

This work is licensed under a Creative Commons Attribution-NonCommercial-NoDerivatives 4.0 International (CC BY-NC-ND 4.0) License. 